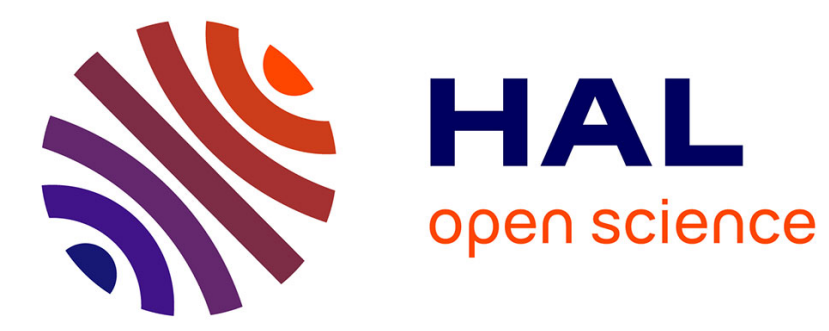

\title{
Microbial Modulation of the Development and Physiology of the Enteric Nervous System
}

François Leulier, Amélie Joly, Filipe de Vadder

\section{To cite this version:}

François Leulier, Amélie Joly, Filipe de Vadder. Microbial Modulation of the Development and Physiology of the Enteric Nervous System. Trends in Microbiology, 2021, 29 (8), pp.686-699. 10.1016/j.tim.2020.11.007 . hal-03376787

\section{HAL Id: hal-03376787 \\ https://hal.science/hal-03376787}

Submitted on 13 Oct 2021

HAL is a multi-disciplinary open access archive for the deposit and dissemination of scientific research documents, whether they are published or not. The documents may come from teaching and research institutions in France or abroad, or from public or private research centers.
L'archive ouverte pluridisciplinaire HAL, est destinée au dépôt et à la diffusion de documents scientifiques de niveau recherche, publiés ou non, émanant des établissements d'enseignement et de recherche français ou étrangers, des laboratoires publics ou privés. 


\title{
Microbial modulation of enteric nervous system development and physiology
}

\author{
Amélie Joly ${ }^{1}$, François Leulier ${ }^{1}$, Filipe De Vadder ${ }^{1, \#}$ \\ ${ }^{1}$ Institut de Génomique Fonctionnelle de Lyon, Université de Lyon, École Normale Supérieure \\ de Lyon, Centre National de la Recherche Scientifique, Université Claude Bernard Lyon 1, \\ UMR5242, Lyon, France.
}

\# To whom correspondence should be addressed: filipe.de_vadder@ens-lyon.fr

\begin{abstract}
The gastrointestinal tract harbors an intrinsic neuronal network, the enteric nervous system (ENS). The ENS controls motility, fluid homeostasis and blood flow, but also interacts with other components of the intestine such as epithelial and immune cells. Recent studies indicate that gut microbiota diversification occurring concomitantly to postnatal ENS maturation could be critical for ENS development and function. Here we discuss the possibility that this functional relationship starts in utero, whereby the maternal microbiota would prime the developing ENS and shape its physiology. We review the ENS/microbiota interactions and their modulation in physiological and pathophysiological contexts. While the microbial modulation of ENS physiology is now well established, further studies are required to understand the contribution of the gut microbiota to the ENS development and pathologies and to reveal the precise mechanisms underlying microbiota-to-ENS communications.
\end{abstract}

Keywords: enteric nervous system/microbiota/enteroendocrine cells/intestinal immune system/intestinal physiology 
3 The gut is a densely innervated organ, with both an extrinsic and intrinsic innervation (the enteric nervous system, ENS). The ENS is a network of different nervous cell types located along the gastrointestinal (GI) tract, from the esophagus extending down to the anus. Because most of its component neurons are not directly connected to the central nervous system (CNS), the ENS represents an independent subdivision of the autonomous nervous system, receiving both sympathetic and parasympathetic outputs [1].

The anatomy of the ENS has been extensively described elsewhere [2]. Briefly, it comprises two ganglionated plexuses (Figure 1): the submucosal plexus, located at the interface of the submucosa and the circular muscle layer, and the myenteric plexus, located between the two muscular layers of the gut. Within each plexus, the neuronal cell bodies form groups called ganglia (Figure 1). Neurons in the ENS can be distinguished by their anatomy, their projections, as well as the different neurotransmitters they secrete. Classic neurochemical coding has shown that the ENS mostly comprises cholinergic excitatory and nitrergic inhibitory motor neurons [2]. However, new molecular biology techniques, particularly single-cell sequencing, have allowed to cluster and redefine new subtypes of enteric neurons, revealing several groups of myenteric neurons in mice and humans [3,4]. Besides neurons, the ENS contains glial cells, which form a unique lineage, distinct from other peripheral and central glia (Figure 1). Glial cells provide nutritional support to neurons but also play an active role in neurotransmission and exert neuroprotective roles on the ENS [2].

Unlike the central nervous system, which is separated from the surrounding environment by a blood-brain barrier, there is no physical separation between the ENS and the surrounding tissues. Thus, the ENS resides in close proximity with muscle cells, stromal cells, interstitial cells of Cajal, epithelial or immune cells [2]. In order to control gut physiology and homeostasis, the ENS requires coordinated regulation among these cell types.

\section{Physiology of the ENS and impact of nutrients}

The first evidence of the autonomous nature of the ENS can be traced back to the $18^{\text {th }}$ century, with the work of Albrecht von Haller. However, it was not until the 1990s that first evidence showed that the ENS contained its own population of sensory neurons and that these neurons 
The ENS provides local control of motility, secretion, absorption, vasodilation and constriction (Figure 1) [6]. The coupling of intestinal secretion and vasodilation allows the movement of water and electrolytes across the epithelium. These functions are mainly regulated by vasomotor and secretomotor neurons from the submucosal plexus, often distinguished by their expression of vasoactive intestinal peptide (VIP) or ChAT [7].

Absorption and secretion are tightly linked to intestinal motility. Digestion of food and absorption of nutrients is conditioned by the presence of chyme content, which travels down the GI through the impulsion of tightly regulated motor movements. After food intake, mechanical and chemical stimuli activate mechanosensitive and intrinsic primary afferent neurons (IPANs) to stimulate motility [8]. In the myenteric plexus, motility reflex arcs induce propulsion through peristalsis and mixing of the chyme through segmentation.

Several nutrients, such as glucose, fatty acids or amino acids indirectly modulate the physiology of the ENS [9]. Indeed, enteric neurons can be activated by GI hormones secreted by enteroendocrine cells (EECs) in response to nutrient detection, e.g. CCK secretion in response to oleate infusion [10] or GLP-1 secretion induced by partial hyperglycemia [11]. The ENS also mediates the effects of nutrients by modulating GI motility, and thus energy availability. For example, application of the short-chain fatty acid (SCFA) butyrate into the colonic mucosa induces peristaltic activity via activation of ENS pathways [12]. Besides modulating neuronal excitability, nutrients also induce changes in the anatomy of the ENS. For example, long-term exposure to high-fat diet induces a reduction in the number of enteric neurons, i.e. enteric neuropathy [13].

\section{In utero development of the ENS: an indirect influence of the microbiota? (Figure 2)}

Most ENS neurons are differentiating during embryonic development in humans [14] and mice [15], i.e. in a context devoid of microbiota. However, evidence show that microbes can influence the foetus development in utero. For example, vaginal dysbiosis linked to Ureaplasma parvum infection induces a loss of enteric neurons and glial cells in ovine fetal ENS [16]. Could maternal microbiota thus affect development of the ENS in utero?

The human GI tract houses a large population of microorganisms that form the gut microbiota. It consists of a diverse community of several hundreds of bacterial species playing a leading role for the host physiology, homeostasis and development [17].

Notably, recent studies showed that the gut microbiota can affect embryonic neuronal development. Indeed, germ-free (GF) mice have altered prenatal transcriptomic profile and 
activation of microglial cells, i.e. resident macrophages of the brain, which are critical for neuronal development [18]. Functional equivalents to brain microglia also exist in the ENS, muscularis macrophages (MMs), which are involved in modulation of enteric neuronal networks (discussed below). Could MMs be influenced by the absence of maternal gut microbiota similarly to their brain counterpart? MMs colonize the fetal mouse bowel before enteric neurons [19], impacting the later ENS development. This possibility raises interesting questions regarding the potential effects of the maternal microbiota on the development of the fetal ENS and on their mediators. Unfortunately, to our knowledge, no study has focused on the developing ENS of GF mice to answer these questions. Interestingly, models of reversible gestational colonization based on the auxotrophic Escherichia coli HA107 strain have been recently elaborated [20]. This kind of experimental setup could be used to investigate the specific influence of the maternal gut microbiota and its modification on the ENS development in utero.

During fetal development, cells derived from the vagal and sacral neural crest colonize the bowel and give rise to enteric glial cells and numerous subtypes of enteric neurons in a sequential manner [21]. Early-born neurons such as serotonergic and cholinergic neurons can be impacted by products ingested by the mother [22]. These neurons influence the future fate of yet undifferentiated neurons. The maternal diet could therefore also impact late neuronal differentiation. Hirschsprung's disease, a life-threatening condition that affects 1 in 5000 infants, arises from a defect in ENS development, which fails to colonize part of or the entire colon [23]. Interestingly, studies in zebrafish have shown that a defect in retinoic acid supplying, which derives from diet-associated vitamin A, leads to a disorganized migration of neural crest cells along the gut and to the occurrence of subsequent aganglionic regions [24]. This observation supports the notion that in utero ENS development is impacted by the maternal diet. Consistently, maternal protein restriction induces a modification of the composition of the ENS and of its functioning in the offspring, with increased propulsive colonic activity [25]. Thus, an unbalanced maternal diet, with concomitant changes in microbiota composition, such as malnutrition or high-fat diet, could also have deleterious consequences in utero.

SCFAs are microbiota-derived metabolites [26] that could potentially be a molecular connection between the maternal microbiota and the fetal ENS development. Indeed, SCFAs produced by the microbiota circulate into the maternal blood and are thought to be able to affect the fetus after placenta crossing [27,28]. In vitro, SCFAs are critical for human neural cell progenitor proliferation [28], suggesting that SCFAs circulating into the fetal blood may impact ENS formation. To our knowledge, the effects of SCFAs on the developing ENS have not been 
studied. A focus on the ENS of pups from mothers fed with a high-fiber diet (inducing production of SCFAs) could help verify this hypothesis.

Bacterial metabolites and by-products activate the secretion of many gut-related hormones by EECs [29], which directly affect the ENS. For example, serotonin (5-HT) derived from maternal enterochromaffin cells, a subset of EECs, is an essential maternal hormone for in utero development [30]. Interestingly, antidepressant treatments that affect the maternal gut microbiota modify 5-HT levels in the mother, which is associated with increased risks of GI diseases development in the offspring [31]. Consistently, dysregulated levels of 5-HT were measured in the mucosa and the myenteric plexus of pups submitted to prenatal alcohol exposure, which also modifies maternal gut microbiota [32].

Through bacterial metabolites and microbiota-regulated signaling pathways, the maternal microbiota could then prenatally participate to the offspring's ENS development in utero.

\section{ENS, microbiota and juvenile life (Table 1)}

ENS development largely extends postnatally [33]. Studies in GF mice showed that very early post-natal alterations (as early as P3), such as a decrease in nerve density and increased proportion of nitrergic neurons, are detected in the ENS [34]. Moreover, alterations of the forebrain development of GF mice, including changes in neuronal death and microglial colonization, already occur a few hours after birth [35]. ENS development is thus likely affected by gut microbiota directly after birth.

The early microbiome of the human infant is highly influenced by the maternal microbiota. Notably, $24 \mathrm{~h}$ after birth, $50 \%$ of the species present in the infant gut microbiota originate from the mother's microbiota [36]. After birth, the infant's microbiota undergoes drastic changes including an increasing diversity and a proliferation of specific phylum. For example, Bifidobacterium are key components of the milk-fed newborn microbiota because they can digest milk-derived oligosaccharides. They are predominant in the human gut microbiota during the first year of life [37]. During the perinatal period, the colonization of the gut by the microbiota is influenced by many factors such as the mode of delivery, gestational age at birth, early-life stress, use of medication and mode of feeding (breast vs formula) [38]. Concomitantly, ENS neurogenesis, maturation and synaptogenesis continues. Notably, during the first weeks of life, the production of late-born neurons is stimulated by the early born serotonergic and cholinergic neurons [22] and the responsiveness of IPANs to 5-HT increases [39]. 
136 Weaning, which occurs approximately 3 weeks after birth in mice and around 6 months of life 137 in humans, drives huge changes in the infant gut microbiota, simultaneously with changes in 138 dietary patterns associated with the interruption of breastfeeding and the passage to solid food. 139 Notably, Bifidobacterium are gradually replaced by Clostridium and Bacteroides, which are 140 more representative of an adult microbiome [37]. These bacteria are able to digest plant-derived 141 oligosaccharides and to produce SCFAs that are essential for the maintenance of a healthy gut 142 [26]. During the same period, the ENS undergoes significant chemical and synaptic remodeling 143 resulting in a maturation of the neuronal network. The number of enteric neurons and glial cells 144 increases in the myenteric plexus and changes in neuronal chemistry occur in the submucosal 145 plexus [40].

146 There is therefore a significant temporal coupling between ENS and microbiota maturation 147 during both perinatal and post-weaning periods in humans and mice. Interestingly, GF adult 148 mice have a newborn-like ENS, with a capacity of plasticity that can be activated by microbiota 149 colonization during adulthood [41]. ENS changes in adolescence could thus be related to 150 changes in the gut microbiota. Moreover, breast milk, which shapes the infant's gut microbiota, 151 also promotes enteric neurogenesis and neuronal growth during the perinatal period [42]. These 152 data suggest the existence of direct and indirect mechanistical links between diet, gut 153 microbiota and ENS development postnatally.

154 Even though several studies have focused on the ENS development in GF mice [41,43,44], 155 reports investigating the effects of antibiotics provide further evidence of the impact of gut 156 microbiota on ENS development. Vancomycin treatment in neonatal mice leads to ENS 157 modifications including neuronal loss, an increase of calbindin-positive neurons, a decrease of 158 nitrergic neurons and a higher speed of colonic contractions [45]. Similarly, antibiotics in 159 adolescent mice also affects the ENS with a decrease of cholinergic neurons, inducing an 160 expected reduction of intestinal motility [46]. It is noteworthy that a similar vancomycin 161 treatment has different functional consequences on neonates and adolescent mice. Indeed, it 162 does not affect the same neuronal subtypes and triggers opposite effects on colonic contractions $163[45,46]$. Moreover, P3 GF mice have an increased proportion of nitrergic neurons [34] whereas 164 P0-P9 vancomycin-treated mice have a decreased proportion of nitrergic neurons [45]. These 165 data suggest that the effects of microbiota on ENS maturation are dependent on the developmental window considered as well as on the nature of the microbiota change.

167 Interestingly, a conditional deletion of choline acetyltransferase (ChAT) in the ENS is related 168 to altered microbial composition in the cecal and colonic microbiota [47]. Because antibiotic 169 treatment induces a decrease of cholinergic neurons in adolescent mice [46], this finding 
indicates a reciprocal effect between the ENS and microbiota composition. Changes in the gut microbiota composition may alter the ENS, thus inducing dysmotility, itself altering the microbial composition.

It is noteworthy that antibiotic treatment during juvenile life alters the expression pattern of brain-derived neurotrophic factor (BDNF), BDNF being a key regulator of ENS development and maturation, this data provides potential insights into the molecular mechanisms between the gut microbiota and the developing postnatal ENS. Similar modifications of BDNF signaling are also observed in mouse models of irritable bowel syndrome (IBS) [48]. IBS is a functional gastrointestinal disorder associated with disturbed defecation and abdominal pain notably due to ENS dysfunction. IBS pathogenesis is thought to be driven by genetic and environmental factors, and several studies have associated the development of pediatric IBS with an altered gut microbiota [49]. The relationship between microbiota, diet and early postnatal ENS development could thus have systemic and/or long-lasting effects impacting energy homeostasis and disease pathogenesis later in life.

\section{Microbial modulation of ENS physiology: the role of enteroendocrine cells (Figure 3)}

As commented before, the gut microbiota plays a crucial role in the development and maturation of the ENS. The relevance of microbiota-ENS interactions is highlighted by abnormalities in morphology and activity of the ENS of GF mice $[34,41,43,50]$. Detection of the gut microbiota, or their metabolites, either directly or indirectly via epithelial cells, is a core sensory system where alterations in microbial composition activate extrinsic sympathetic gutprojecting neurons [51]. GF mice exhibit delayed transit time, while re-colonization restores transit time and innervation of the mucosa [41].

Microbial factors may act through Toll-Like Receptors (TLRs), which are expressed in epithelial cells [52], glia [53] and neurons [54,55] and specifically recognize pathogenassociated molecular patterns (PAMPs). For example, TLR2 (a receptor that recognizes lipoproteins of the bacteria cell wall) signaling regulates inflammation by controlling ENS structure along with intestinal neuromuscular function [55]. Furthermore, in the adult mouse colon, TLR2 promotes neurogenesis after antibiotic treatment [56]. However, the fact that TLRs are expressed by several types of cells in the intestine makes difficult to identify the specific cells connecting the signaling between microbiota and the ENS.

Two recent studies gave further hints of the molecular coupling between gut microbiota and the ENS. Obata et al. showed that most neurons of the distal gastrointestinal tract express Aryl 
Hydrocarbon Receptor, a nuclear receptor activated by several tryptophan-derived metabolites.

205 The gut microbiota was able to induce expression of this receptor in these neurons, increasing intestinal motility, among other patterns [50]. Chandrasekaran et al. studied the impact of the widely used probiotic Lactobacillus rhamnosus GG and found that its presence in the GI tract stimulated neuronal differentiation of cholinergic neurons and, in fine, intestinal motility [57]. How do gut microbes communicate with the host to modulate physiology of the ENS? As mentioned before, there is no direct detection of luminal contents by components of the ENS. The first line of detection of such contents are epithelial cells, which are in direct contact with the gut lumen. Thus, interactions between neurons or glia and epithelial cells represent a key mechanism of transmission of microbiota-mediated cues to the ENS [29]. Amongst gut epithelial cells, enteroendocrine cells (EECs) of the gut barely represent 1\% of the epithelium, yet are essential to intestinal physiology [58]. Increasing evidence shows that connections between enteric neurons and EECs regulate gut physiology. Recent work shows that some EECs termed "neuropod cells" directly communicate with neurons through modified synapses [59]. For example, a specific set of EECs, enterochromaffin cells, secretes 5-HT in response to a wide variety of microbiota-derived chemicals in the colonic lumen [60,61]. Single-cell RNA sequencing of enteric neurons shows that they express receptors of several gut hormones, including HTR3a, HTR4 (5-HT receptors) and could be therefore able to respond to enterochromaffin cells activation [3]. Since the gut epithelium is renewed once every seven days, this raises the question of the dynamics and plasticity of these synapses. Indeed, the ENS is involved in the proliferation of the intestinal epithelium. Muscarinic acetylcholine (Ach) receptors (named M1 to M5) are widely expressed in the epithelium and knockout of either receptor M2, M3 or M5 increases intestinal proliferation [62], associated to the activation of 5HT2A receptors on cholinergic neurons [63]. Furthermore, in vitro co-culturing of intestinal stem cells with enteric neurons and glia promotes epithelial proliferation [64] and differentiation into EECs [65].

230 Enterochromaffin cells in the gut can act as mechanosensors, releasing large quantities of 5-HT 231 in response to mechanic distension [66-68]. 5-HT secreted from the EECs could directly impact enteric neurons. Indeed, colonization of GF Tphl-deficient mice (i.e. mice with no production of 5-HT in the epithelium) results in decreased number of myenteric neurons, showing that EEC-derived 5-HT is crucial for the integrity of the ENS after colonization with gut microbiota [41].

How can 5-HT secretion be directly linked to gut microbes and regulation of intestinal 
[69]. While 5-HT is mostly released from the basal side, several studies have found that EECderived 5-HT can end up in the gut lumen and feces $[69,70]$. A recent study found that 5-HT promotes intestinal colonization of the spore-forming gut bacterium Turicibacter sanguinis [71]. Interestingly, both $T$. sanguinis and mucosal 5-HT have been found to be strongly proinflammatory [72-74], a state that is classically linked to reduced motility of the intestine [75]. However, on the basal side, 5-HT has been described as a promoter of motility through interactions with the ENS. 5-HT can activate the terminals of primary sensory neurons [76], but recent data have challenged the concept that 5-HT is the initiator of motor activity in the colon (widely discussed in reference [5]). Indeed, a transcriptomic analysis of enterochromaffin cells of the small intestine has shown that these cells lack $G$ protein-coupled receptors for lipid and protein metabolites but highly express Glplr in the basal side [60]. GLP-1 induces 5-HT release in vitro [60]. Thus, L cells, a class of EECs which produce GLP-1 in response to nutrients [60], could act as a mediator between nutrient sensing and 5-HT release to stimulate intestinal motility in the small intestine.

252 Could it be that GLP-1 exerts opposite effects on intestinal motility in the colon and in the small intestine? GF mice, which have reduced gastrointestinal motility, have elevated levels of GLP1 [77]. GF mice have reduced capacity of energy harvest [78] and thus the energy status of their colon may be assimilated to chronic undernutrition. In such conditions, GLP-1 induces slower transit time [77]. Thus, we can hypothesize that transit slowing is a physiological adaptation to chronic undernutrition triggered by nutrient scarcity and an altered microbiota in order to increase energy harvest and compensate for the reduced availability of nutrients in the lumen. The aim now is to understand the intricate microbiota/EEC/ENS relationship and its involvement in pathophysiology of diseases associated to impaired intestinal function.

Modulation of host physiology by the ENS and microbiota-neuroimmune interactions (Figure 4)

The intestinal immune system is crucial in maintaining homeostasis of the gut. Interactions between the ENS and immune cells have been thoroughly described as master regulators of gut physiology [79].

In the context of immune-mediated regulation of gut motility, muscularis macrophages (MMs) residing in close contact to the myenteric plexus have been shown to modulate peristalsis by secretion of bone-derived morphogenetic protein 2 (BMP2), stimulating enteric neurons, which 
addition, signals derived from the gut microbiota modulate the levels of both BMP2 and CSF1, tuning the interaction between enteric neurons and macrophages. However, other cell types are also involved in fine-tuning interactions between microbiota and the ENS. Type-3 innate lymphoid cells (ILC3) promote intestinal and metabolic homeostasis by integrating cytokinederived signals [81]. ILC3 secrete interleukin 22 (IL-22), which promotes the production of antimicrobial peptides and mucin in the epithelium [82]. These cells express VIP receptor 2 (VPAC2) and lie in close proximity to VIPergic neurons in the lamina propria [83]. In response to feeding, particularly in the presence of fat in the luminal content, VIPergic neurons antagonize microbiota-induced secretion of IL-22 by ILC3, increasing lipid absorption and thus maintaining a trade-off between immune protection and the efficiency of nutrient absorption [83]. Modulation of microbiota-immune interactions may therefore be effective for the treatment of metabolic diseases, which are usually associated to inflammation in the gut. Indeed, during metabolic diseases such as type 2 diabetes, intestinal inflammation is associated with dysfunction of enteric neurons [84]. Analysis of lamina propria and MMs showed that the latter mainly expressed M2-type wound-healing and anti-inflammatory genes [85]. Moreover, MMs express $\beta 2$ adrenergic receptors and reside in close proximity to enteric neurons. Infection with Salmonella typhimurium spiB activates extrinsic noradrenergic neurons, increasing transit time [85] and resulting in neuronal loss in the ENS [86]. By upregulating signaling via $\beta 2$ adrenergic receptors, MMs respond to infection and induce a neuroprotective effect in the ENS, limiting infection-induced neuronal damage [86].

Extrinsic nociceptor neurons innervating Peyer patches have been shown to play a critical role in protecting against infection with Salmonella typhimurium [87]. However, intrinsic innervation also plays a role in host defense against pathogens. Particularly, enteric neuronderived interleukin-18 regulates the production of antimicrobial peptides in the epithelium, targeting pathogens such as S. typhimurium [88].

Thus, immune cells also play a major role in coupling microbial signals from the gut lumen to enteric neurons.

In addition to its role in sensing nutrients (as discussed before), a recent study showed that the ENS also plays a crucial role in regulating glucose homeostasis. Muller, Matheis, Schneeberger et al. studied the particular role of cocaine- and amphetamine-regulated transcript (CART)expressing neurons of the ENS. The number of CART-expressing neurons was increased by 2fold in mice with a gut microbiota, when compared to GF mice. Furthermore, CART neurons were characterized as viscerofugal (i.e. with axons projecting to the viscera) and were polysynaptically connected to the liver and pancreas via the celiac and superior mesenteric 
ganglia. Specific activation of CART in the ENS led to increased blood glucose and decreased insulin, while specific ablation of the neurons resulted in decreased blood glucose, increased insulin and decreased gluconeogenesis in the liver [89]. Overall, this study showed that specific neurons from the ENS can directly regulate blood glucose without the need of any input from the CNS.

\section{Conclusions and perspectives}

It is now well established that environmental signals that modulate the gut microbiota lead to persistent changes in the development and physiology of the ENS. Enteric neurons and glial cells' response to microbial cues are key drivers of these physiological adaptations. However, the specific molecular and cellular paths that drive the changes in the ENS development and function remain to be elucidated. Particularly, the scientific literature is lacking data on how the maternal microbial environment shapes the development of the ENS in utero and if these changes have long-lasting effect on the physiology and physiopathology of the GI tract. Furthermore, research needs to focus on the specific role of the ENS in maintaining energy homeostasis. The capacity of enteric neurons to respond to food-derived signals suggests that the ENS could be an important actor, along gut-brain communications, in the preservation of the energy balance. Finally, recent advances in imaging and single-cell sequencing technologies have greatly benefited the study of enteric neuron circuits and helped identify neurons subtypes owning a specific machinery to detect nutrients and microbiota-derived signals. Combining these advances with genetic manipulations and physiological analysis will allow to functionally dissect the contribution of these specific enteric neuronal subtypes to the modulation of intestinal development and physiology by microbes.

\section{Competing interests}

332 The authors declare no conflict of interest in relation to this publication.

\section{Acknowledgements}

335 The authors would like to thank Anne Lambert (Institut de Génomique Fonctionnelle de Lyon) 336 for her help in obtaining images in Figure 1. F.D.V. is supported by a grant from La Fondation 337 des Treilles. A.J. is supported by a doctoral grant from the French Ministry of Research. 338 Research in F.L lab is supported by Université de Lyon, ENS de Lyon and CNRS and funded 
339 by an FRM grant (Équipe FRM DEQ20180339196) and an ANR grant (ANR-18-CE15-0011340 01). 


\section{References}

1 Uesaka, T. et al. (2016) Development of the intrinsic and extrinsic innervation of the gut. Dev. Biol. 417, $158-167$

2 Fung, C. and Vanden Berghe, P. (2020) Functional circuits and signal processing in the enteric nervous system. Cell. Mol. Life Sci. DOI: 10.1007/s00018-020-03543-6

3 Zeisel, A. et al. (2018) Molecular Architecture of the Mouse Nervous System. Cell 174, 999-1014.e22

4 Drokhlyansky, E. et al. (2020) The Human and Mouse Enteric Nervous System at Single-Cell Resolution. Cell DOI: $10.1016 /$ j.cell.2020.08.003

5 Spencer, N.J. and $\mathrm{Hu}, \mathrm{H}$. (2020) Enteric nervous system: sensory transduction, neural circuits and gastrointestinal motility. Nat Rev Gastroenterol Hepatol 17, 338-351

6 Furness, J.B. (2012) The enteric nervous system and neurogastroenterology. Nat Rev Gastroenterol Hepatol 9, 286-294

7 Foong, J.P.P. et al. (2014) Properties of cholinergic and non-cholinergic submucosal neurons along the mouse colon. J. Physiol. (Lond.) 592, 777-793

8 Lai, N.Y. et al. (2017) Sensory neuron regulation of gastrointestinal inflammation and bacterial host defence. J. Intern. Med. 282, 5-23

9 Neunlist, M. and Schemann, M. (2014) Nutrient-induced changes in the phenotype and function of the enteric nervous system. J. Physiol. (Lond.) 592, 2959-2965

10 Gulley, S. et al. (2005) Cholecystokinin1 receptors mediate the increase in Fos-like immunoreactivity in the rat myenteric plexus following intestinal oleate infusion. Physiol. Behav. 86, 128-135

11 Richards, P. et al. (2014) Identification and characterization of GLP-1 receptor-expressing cells using a new transgenic mouse model. Diabetes 63, 1224-1233

12 Grider, J.R. and Piland, B.E. (2007) The peristaltic reflex induced by short-chain fatty acids is mediated by sequential release of 5-HT and neuronal CGRP but not BDNF. Am. J. Physiol. Gastrointest. Liver Physiol. 292, G429-437

13 Grasset, E. et al. (2017) A Specific Gut Microbiota Dysbiosis of Type 2 Diabetic Mice Induces GLP-1 Resistance through an Enteric NO-Dependent and Gut-Brain Axis Mechanism. Cell Metab. 25, 1075-1090.e5

14 McCann, C.J. et al. (2019) Neuronal Development and Onset of Electrical Activity in the Human Enteric Nervous System. Gastroenterology 156, 1483-1495.e6

15 Memic, F. et al. (2018) Transcription and Signaling Regulators in Developing Neuronal Subtypes of Mouse and Human Enteric Nervous System. Gastroenterology 154, 624-636

16 Heymans, C. et al. (2020) Chronic Intra-Uterine Ureaplasma parvum Infection Induces Injury of the Enteric Nervous System in Ovine Fetuses. Front Immunol 11, 189

17 Schroeder, B.O. and Bäckhed, F. (2016) Signals from the gut microbiota to distant organs in physiology and disease. Nat. Med. 22, 1079-1089

18 Thion, M.S. et al. (2018) Microbiome Influences Prenatal and Adult Microglia in a Sex-Specific Manner. Cell 172, 500-516.e16

19 Avetisyan, M. et al. (2018) Muscularis macrophage development in the absence of an enteric nervous system. Proc. Natl. Acad. Sci. U.S.A. 115, 4696-4701

20 Cuenca, M. et al. (2016) D-Alanine-Controlled Transient Intestinal Mono-Colonization with NonLaboratory-Adapted Commensal E. coli Strain HS. PLoS ONE 11, e0151872

21 Bergner, A.J. et al. (2014) Birthdating of myenteric neuron subtypes in the small intestine of the mouse. $J$. Comp. Neurol. 522, 514-527

22 Rao, M. and Gershon, M.D. (2018) Enteric nervous system development: what could possibly go wrong? Nat. Rev. Neurosci. 19, 552-565

23 Heuckeroth, R.O. (2018) Hirschsprung disease - integrating basic science and clinical medicine to improve outcomes. Nat Rev Gastroenterol Hepatol 15, 152-167

24 Uribe, R.A. et al. (2018) Retinoic acid temporally orchestrates colonization of the gut by vagal neural crest cells. Dev. Biol. 433, 17-32

25 Aubert, P. et al. (2019) Maternal protein restriction induces gastrointestinal dysfunction and enteric nervous system remodeling in rat offspring. FASEB J. 33, 770-781

26 Koh, A. et al. (2016) From Dietary Fiber to Host Physiology: Short-Chain Fatty Acids as Key Bacterial Metabolites. Cell 165, 1332-1345

27 Hsu, C.-N. et al. (2018) Maternal Administration of Probiotic or Prebiotic Prevents Male Adult Rat Offspring against Developmental Programming of Hypertension Induced by High Fructose Consumption in Pregnancy and Lactation. Nutrients 10 ,

28 Yang, L.L. et al. (2019) Enteric short-chain fatty acids promote proliferation of human neural progenitor cells. J. Neurochem. DOI: $10.1111 /$ jnc. 14928

29 Walsh, K.T. and Zemper, A.E. (2019) The Enteric Nervous System for Epithelial Researchers: Basic Anatomy, Techniques, and Interactions With the Epithelium. Cell Mol Gastroenterol Hepatol 8, 369-378 
30 Kliman, H.J. et al. (2018) Pathway of Maternal Serotonin to the Human Embryo and Fetus. Endocrinology $159,1609-1629$

31 Salisbury, A.L. et al. (2020) Prenatal antidepressant exposures and gastrointestinal complaints in childhood: A gut-brain axis connection? Dev Psychobiol 62, 816-828

32 Dylag, K.A. et al. (2018) Prenatal alcohol exposure reduces 5-HT concentration in mouse intestinal muscle and mucosa. Environ. Toxicol. Pharmacol. 61, 24-29

33 Foong, J.P.P. (2016) Postnatal Development of the Mouse Enteric Nervous System. Adv. Exp. Med. Biol. 891, 135-143

34 Collins, J. et al. (2014) Intestinal microbiota influence the early postnatal development of the enteric nervous system. Neurogastroenterol. Motil. 26, 98-107

35 Erny, D. et al. (2015) Host microbiota constantly control maturation and function of microglia in the CNS. Nat. Neurosci. 18, 965-977

36 Ferretti, P. et al. (2018) Mother-to-Infant Microbial Transmission from Different Body Sites Shapes the Developing Infant Gut Microbiome. Cell Host Microbe 24, 133-145.e5

37 Bäckhed, F. et al. (2015) Dynamics and Stabilization of the Human Gut Microbiome during the First Year of Life. Cell Host Microbe 17, 690-703

38 Fouhy, F. et al. (2019) Perinatal factors affect the gut microbiota up to four years after birth. Nat Commun 10,1517

39 Hao, M.M. et al. (2020) Development of the intrinsic innervation of the small bowel mucosa and villi. American Journal of Physiology-Gastrointestinal and Liver Physiology 318, G53-G65

40 Parathan, P. et al. (2020) The enteric nervous system undergoes significant chemical and synaptic maturation during adolescence in mice. Dev. Biol. 458, 75-87

41 De Vadder, F. et al. (2018) Gut microbiota regulates maturation of the adult enteric nervous system via enteric serotonin networks. Proc. Natl. Acad. Sci. U.S.A. 115, 6458-6463

42 Fichter, M. et al. (2011) Breast milk contains relevant neurotrophic factors and cytokines for enteric nervous system development. Mol Nutr Food Res 55, 1592-1596

43 Kabouridis, P.S. et al. (2015) Microbiota Controls the Homeostasis of Glial Cells in the Gut Lamina Propria. Neuron 85, 289-295

44 McVey Neufeld, K.A. et al. (2015) The gut microbiome restores intrinsic and extrinsic nerve function in germ-free mice accompanied by changes in calbindin. Neurogastroenterol. Motil. 27, 627-636

45 Hung, L.Y. et al. (2019) Neonatal Antibiotics Disrupt Motility and Enteric Neural Circuits in Mouse Colon. Cell Mol Gastroenterol Hepatol 8, 298-300.e6

46 Hung, L.Y. et al. (2020) Antibiotic exposure post weaning disrupts the neurochemistry and function of enteric neurons mediating colonic motor activity. Am. J. Physiol. Gastrointest. Liver Physiol. DOI: 10.1152/ajpgi.00088.2020

47 Johnson, C.D. et al. (2018) Deletion of choline acetyltransferase in enteric neurons results in postnatal intestinal dysmotility and dysbiosis. FASEB J. 32, 4744-4752

48 Bistoletti, M. et al. (2019) Antibiotic treatment-induced dysbiosis differently affects BDNF and TrkB expression in the brain and in the gut of juvenile mice. PLoS ONE 14, e0212856

49 Collins, S.M. (2014) A role for the gut microbiota in IBS. Nat Rev Gastroenterol Hepatol 11, 497-505

50 Obata, Y. et al. (2020) Neuronal programming by microbiota regulates intestinal physiology. Nature 578, 284-289

51 Muller, P.A. et al. (2020) Microbiota modulate sympathetic neurons via a gut-brain circuit. Nature 583, 441446

52 Haber, A.L. et al. (2017) A single-cell survey of the small intestinal epithelium. Nature 551, 333-339

53 Esposito, G. et al. (2014) Palmitoylethanolamide improves colon inflammation through an enteric glia/toll like receptor 4-dependent PPAR- $\alpha$ activation. Gut 63, 1300-1312

54 Barajon, I. et al. (2009) Toll-like receptors 3, 4, and 7 are expressed in the enteric nervous system and dorsal root ganglia. J. Histochem. Cytochem. 57, 1013-1023

55 Brun, P. et al. (2013) Toll-like receptor 2 regulates intestinal inflammation by controlling integrity of the enteric nervous system. Gastroenterology 145, 1323-1333

56 Yarandi, S.S. et al. (2020) Intestinal Bacteria Maintain Adult Enteric Nervous System and Nitrergic Neurons via Toll-like Receptor 2-induced Neurogenesis in Mice. Gastroenterology DOI: 10.1053/j.gastro.2020.03.050

57 Chandrasekharan, B. et al. (2019) Interactions Between Commensal Bacteria and Enteric Neurons, via FPR1 Induction of ROS, Increase Gastrointestinal Motility in Mice. Gastroenterology 157, 179-192.e2

58 Gribble, F.M. and Reimann, F. (2019) Function and mechanisms of enteroendocrine cells and gut hormones in metabolism. Nat Rev Endocrinol 15, 226-237

59 Kaelberer, M.M. et al. (2018) A gut-brain neural circuit for nutrient sensory transduction. Science 361,

60 Lund, M.L. et al. (2018) Enterochromaffin 5-HT cells - A major target for GLP-1 and gut microbial metabolites. Mol Metab 11, 70-83 
61 Bellono, N.W. et al. (2017) Enterochromaffin Cells Are Gut Chemosensors that Couple to Sensory Neural Pathways. Cell 170, 185-198.e16

62 Greig, C.J. and Cowles, R.A. (2017) Muscarinic acetylcholine receptors participate in small intestinal mucosal homeostasis. J. Pediatr. Surg. 52, 1031-1034

63 Gross, E.R. et al. (2012) Neuronal serotonin regulates growth of the intestinal mucosa in mice. Gastroenterology 143, 408-417.e2

64 Workman, M.J. et al. (2017) Engineered human pluripotent-stem-cell-derived intestinal tissues with a functional enteric nervous system. Nat. Med. 23, 49-59

65 Puzan, M. et al. (2018) Enteric Nervous System Regulation of Intestinal Stem Cell Differentiation and Epithelial Monolayer Function. Sci Rep 8, 6313

66 Wang, F. et al. (2017) Mechanosensitive ion channel Piezo2 is important for enterochromaffin cell response to mechanical forces. J. Physiol. (Lond.) 595, 79-91

67 Alcaino, C. et al. (2018) A population of gut epithelial enterochromaffin cells is mechanosensitive and requires Piezo2 to convert force into serotonin release. Proc. Natl. Acad. Sci. U.S.A. 115, E7632-E7641

68 Bertrand, P.P. (2006) Real-time measurement of serotonin release and motility in guinea pig ileum. J. Physiol. (Lond.) 577, 689-704

69 Yano, J.M. et al. (2015) Indigenous bacteria from the gut microbiota regulate host serotonin biosynthesis. Cell 161, 264-276

70 Reigstad, C.S. et al. (2015) Gut microbes promote colonic serotonin production through an effect of shortchain fatty acids on enterochromaffin cells. FASEB J. 29, 1395-1403

71 Fung, T.C. et al. (2019) Intestinal serotonin and fluoxetine exposure modulate bacterial colonization in the gut. Nat Microbiol 4, 2064-2073

72 Goodrich, J.K. et al. (2016) Genetic Determinants of the Gut Microbiome in UK Twins. Cell Host Microbe $19,731-743$

73 Margolis, K.G. et al. (2014) Pharmacological reduction of mucosal but not neuronal serotonin opposes inflammation in mouse intestine. Gut 63, 928-937

74 Ghia, J.-E. et al. (2009) Serotonin has a key role in pathogenesis of experimental colitis. Gastroenterology $137,1649-1660$

75 Sharkey, K.A. et al. (2018) Neuroimmunophysiology of the gut: advances and emerging concepts focusing on the epithelium. Nat Rev Gastroenterol Hepatol 15, 765-784

76 Bertrand, P.P. et al. (1997) Analysis of the responses of myenteric neurons in the small intestine to chemical stimulation of the mucosa. Am. J. Physiol. 273, G422-435

77 Wichmann, A. et al. (2013) Microbial modulation of energy availability in the colon regulates intestinal transit. Cell Host Microbe 14, 582-590

78 Bäckhed, F. et al. (2004) The gut microbiota as an environmental factor that regulates fat storage. Proc. Natl. Acad. Sci. U.S.A. 101, 15718-15723

79 Verheijden, S. and Boeckxstaens, G.E. (2018) Neuroimmune interaction and the regulation of intestinal immune homeostasis. Am. J. Physiol. Gastrointest. Liver Physiol. 314, G75-G80

80 Muller, P.A. et al. (2014) Crosstalk between Muscularis Macrophages and Enteric Neurons Regulates Gastrointestinal Motility. Cell 158, 300-313

81 Spits, H. and Cupedo, T. (2012) Innate lymphoid cells: emerging insights in development, lineage relationships, and function. Annu. Rev. Immunol. 30, 647-675

82 Hooper, L.V. et al. (2012) Interactions between the microbiota and the immune system. Science 336, 12681273

83 Talbot, J. et al. (2020) Feeding-dependent VIP neuron-ILC3 circuit regulates the intestinal barrier. Nature $579,575-580$

84 Abot, A. et al. (2018) Galanin enhances systemic glucose metabolism through enteric Nitric Oxide Synthaseexpressed neurons. Mol Metab 10, 100-108

85 Gabanyi, I. et al. (2016) Neuro-immune Interactions Drive Tissue Programming in Intestinal Macrophages. Cell 164, 378-391

86 Matheis, F. et al. (2020) Adrenergic Signaling in Muscularis Macrophages Limits Infection-Induced Neuronal Loss. Cell 180, 64-78.e16

87 Lai, N.Y. et al. (2020) Gut-Innervating Nociceptor Neurons Regulate Peyer's Patch Microfold Cells and SFB Levels to Mediate Salmonella Host Defense. Cell 180, 33-49.e22

88 Jarret, A. et al. (2020) Enteric Nervous System-Derived IL-18 Orchestrates Mucosal Barrier Immunity. Cell 180, 50-63.e12

89 Muller, P.A. et al. (2020) Microbiota-modulated CART+ enteric neurons autonomously regulate blood glucose. Science DOI: 10.1126/science.abd6176 


\section{Figure legends}

Figure 1. Anatomy of the Enteric Nervous System. (A) Cartoon depiction of the intestinal epithelium with the different layers and cell types of the ENS. The ENS contains several types of neurons, which ensure the regulation of various functions of the intestine (in italic capital letters). Intrinsic primary afferent neurons (IPANs) are activated by cues coming from the mucosa and activate ascending and descending interneurons, which stimulate inhibitory and excitatory motor neurons, as well as secretomotor neurons. Arrows indicate the sense of propagation of the signal. Ach: acetylcholine; Enk: enkephalin; NO: nitric oxyde; NMU: neuromedin U; VIP: vasoactive intestinal peptide; 5-HT: serotonin. (B) Confocal image of a $100 \mu \mathrm{m}$-thick intestinal roll showing structure of the intestine (in blue DAPI stains nuclei) and its very dense innervation that goes from the crypt to the tip of the villus (red, neuron-specific tubulin TuJ1). (C) Magnification of a myenteric ganglion showing the network of (top) glial cells (green, stained with S100ß) and (bottom) neurons (green: pan-neuronal marker HuC/D; blue: neuron-specific tubulin TuJ1; green: inhibitory motor neuron marker nitric oxide synthase 1, NOS1).

Created with Biorender.com

Figure 2. The enteric nervous system (ENS) and the gut microbiota develop concomitantly and in close interaction during juvenile life and are affected by maternal and extrinsic factors. The development of the ENS (blue square) starts during prenatal life when the embryonic gut is colonized by cells derived from the vagal and sacral neural crest cells mainly. The first enteric neurons (early born neurons including serotoninergic and cholinergic neurons) subsequently differentiate. ENS prenatal development is likely influenced by the maternal gut microbiota (orange square), itself shaped by the mother's diet and external cues including the use of antibiotics or xenobiotics. ENS development continues after birth with notably an intense neurogenesis, synaptogenesis and maturation during the first weeks of life. It also extends after weaning with synaptic and neuronal chemistry maturation. During the same time, the infant microbiota matures (Yellow square). For example, breast-fed neonates display a high prevalence of bacteria from the Bifidobacterium phylum. Bacterial diversity increases drastically, notably after weaning and the concurrent shift to solid food. The infant's microbiota composition depends on the mode of feeding and of delivery, the gestational age at birth, the use of antibiotics... (Green square). These factors also influence juvenile ENS development which suggests a strong influence of microbiota on ENS development. The mechanisms underlying microbiota effects on ENS development remain to be investigated. 
Figure 3. Crosstalk between microbiota and epithelial cells regulates ENS physiology. Enteric neurons can communicate with epithelial cells through paracrine ways or through enteroendocrine cells (EECs) with modified synapses, termed "neuropod cells". Microbiotaderived cues (such as lipoproteins from the bacterial cell wall and tryptophan-derived metabolites) activate Toll-like Receptor 2 (TLR2) and aryl hydrocarbon receptor (AHR). Gut microbiota also modulates the production of several gut hormones, such as glucagon-like peptide 1 (GLP-1) and serotonin (5-HT). In enterochromaffin cells, spore-forming bacteria upregulate the expression of tryptophan hydroxylase 1 (TPH1), the rate-limiting enzyme of 5HT synthesis. In turn, 5-HT stimulates growth of spore-forming bacteria and gut motility through activation of HT4 receptor (HT4R). Furthermore, secretion of acetylcholine (Ach) by cholinergic neurons activates epithelial proliferation through several muscarinic receptors.

Created with Biorender.com

Figure 4. Microbiota-immune interactions and the ENS regulate host physiology. Muscularis macrophages (MMs), which reside in the muscularis layer, in close contact to myenteric neurons, secrete Bone Morphogenetic Protein 2 (BMP2), activating BMP2 receptor (BMP2R) in enteric neurons. In response, enteric neurons secrete Colony-Stimulating Factor 1 (CSF1), which binds CSF1 receptor (CSF1R) in macrophages. These interactions are activated by gut microbiota and help maintain gut motility. Salmonella infection leads to neuron loss in the ENS. In order to respond to the infection, extrinsic neurons secrete noradrenaline, activating $\beta_{2}$ adrenergic receptor $\left(\beta_{2} \mathrm{AR}\right)$, preventing neuron loss. Furthermore, enteric neurons respond to Salmonella infection through secretion of interleukin IL-18, activating goblet cells, which secrete antimicrobial peptides (AMPs).

Food intake (and particularly fat) activates VIPergic neurons, which secrete vasoactive intestinal peptide (VIP) in response. VIP activates VIP receptor (VPAC2) in type-3 innate lymphoid cells (ILC3). Production of interleukin 22 (IL-22)), which is regulated by segmented filamentous bacteria, is inhibited by activation of VPAC2, increasing expression of lipid transporters, promoting fat absorption.

The gut microbiota stimulates the expression of cocaine- and amphetamine-related transcript (CART) in viscerofugal enteric neurons. These neurons project to the celiac and superior mesenteric ganglia (CG-SMG), which in turn innervate the liver and pancreas. Activation of CART neurons results in increased blood glucose and decreased insulin levels, showing that these enteric neurons are glucoregulatory. 
Created with Biorender.com

Table 1. Studies focusing on the consequences of microbiota modification on the enteric nervous system (ENS) development during juvenile life. 
A
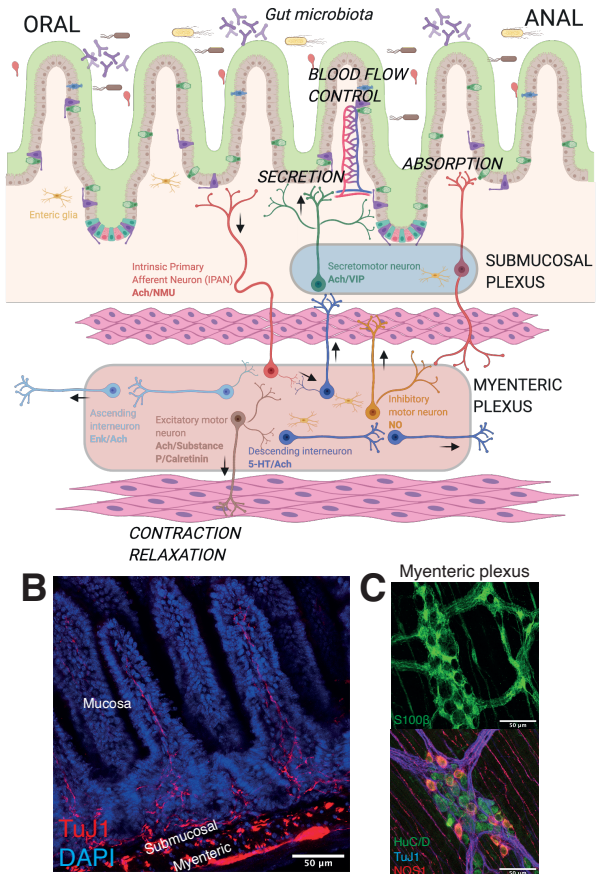

Myenteric plexus

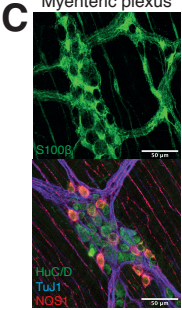


External parameters

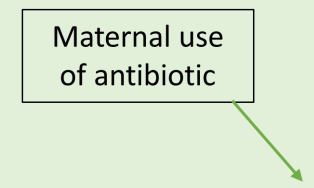

Maternal influence
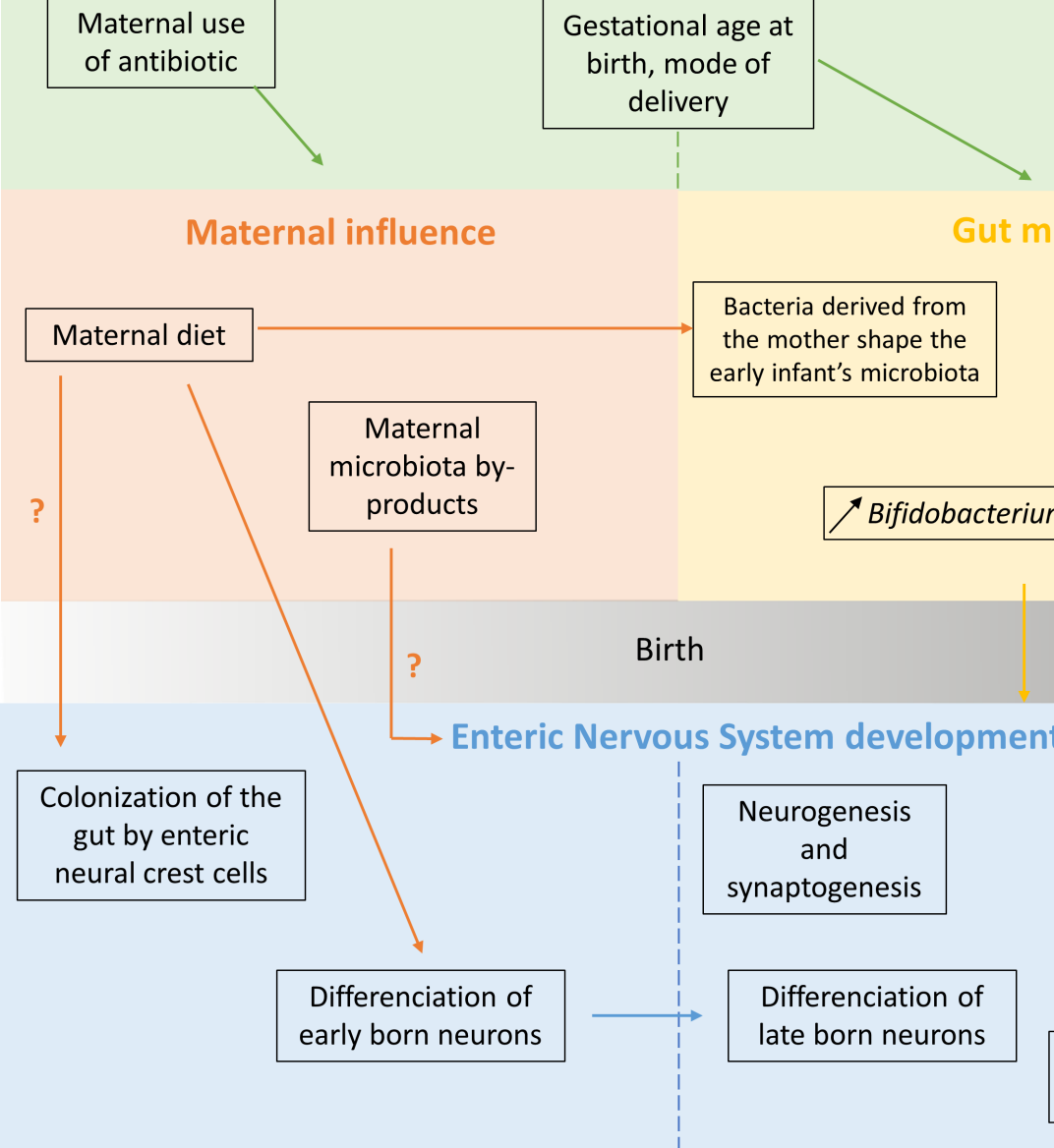

irth

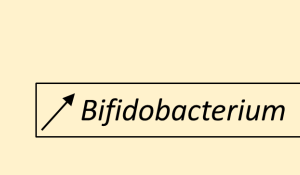

Bifidobacterium
Bacteria derived from the mother shape the early infant's microbiota

Gut microbiota development
Pathological conditions

(e.g. antibiotic treatment, early life stress)

Diet,
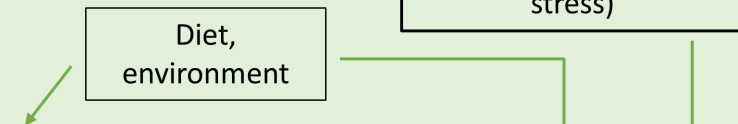

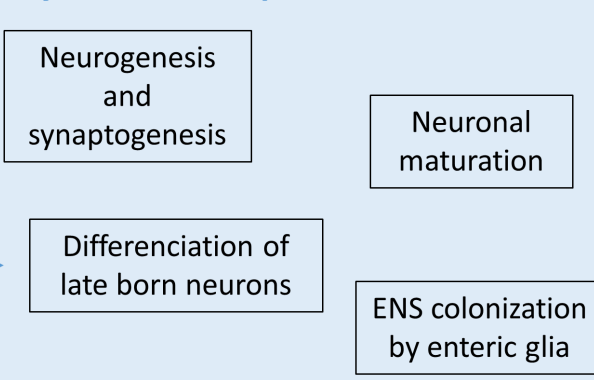

ENS priming for later diseases?

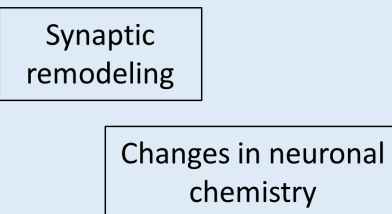
chemistry

Neuro- and glio-genesis 

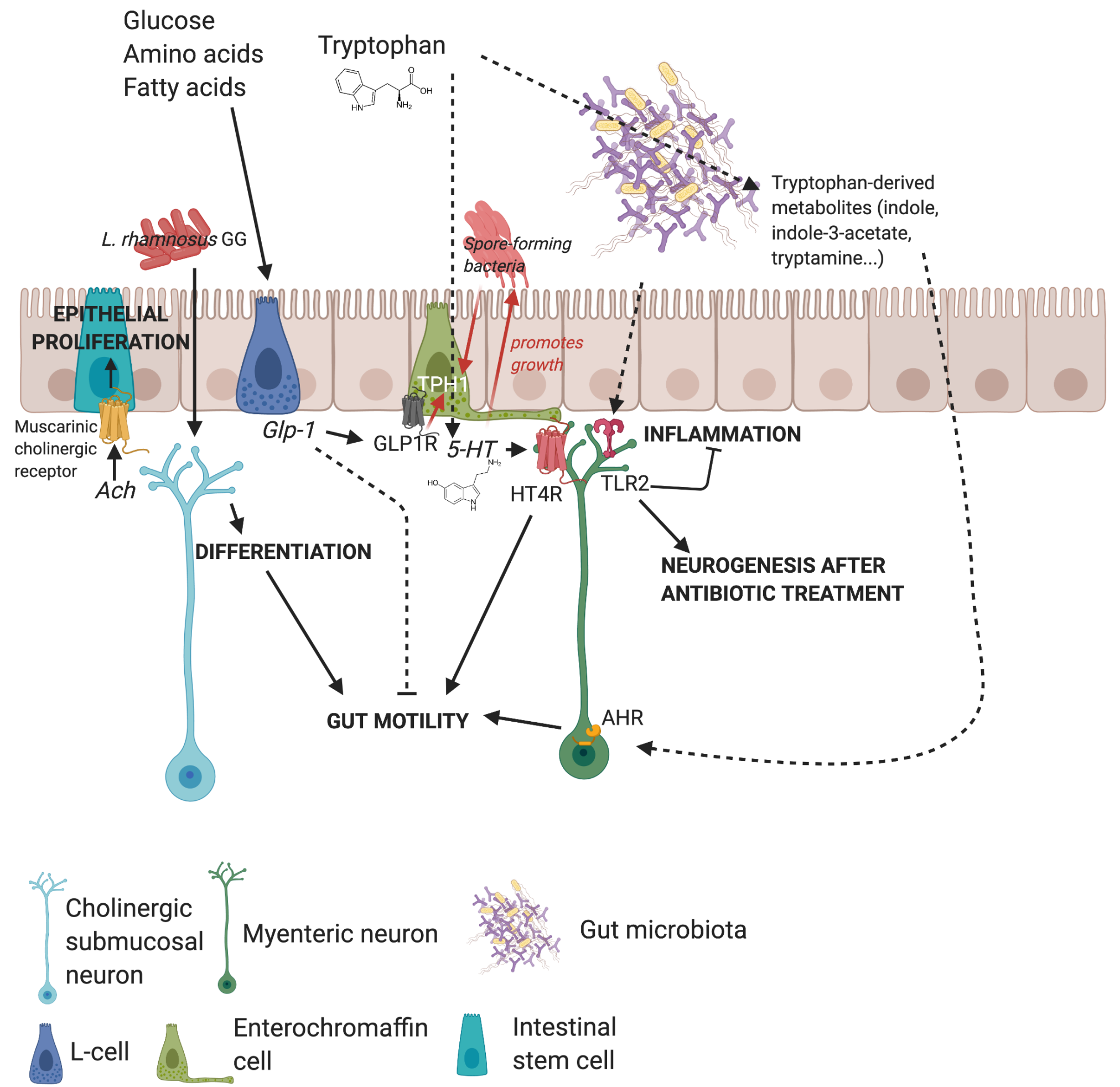


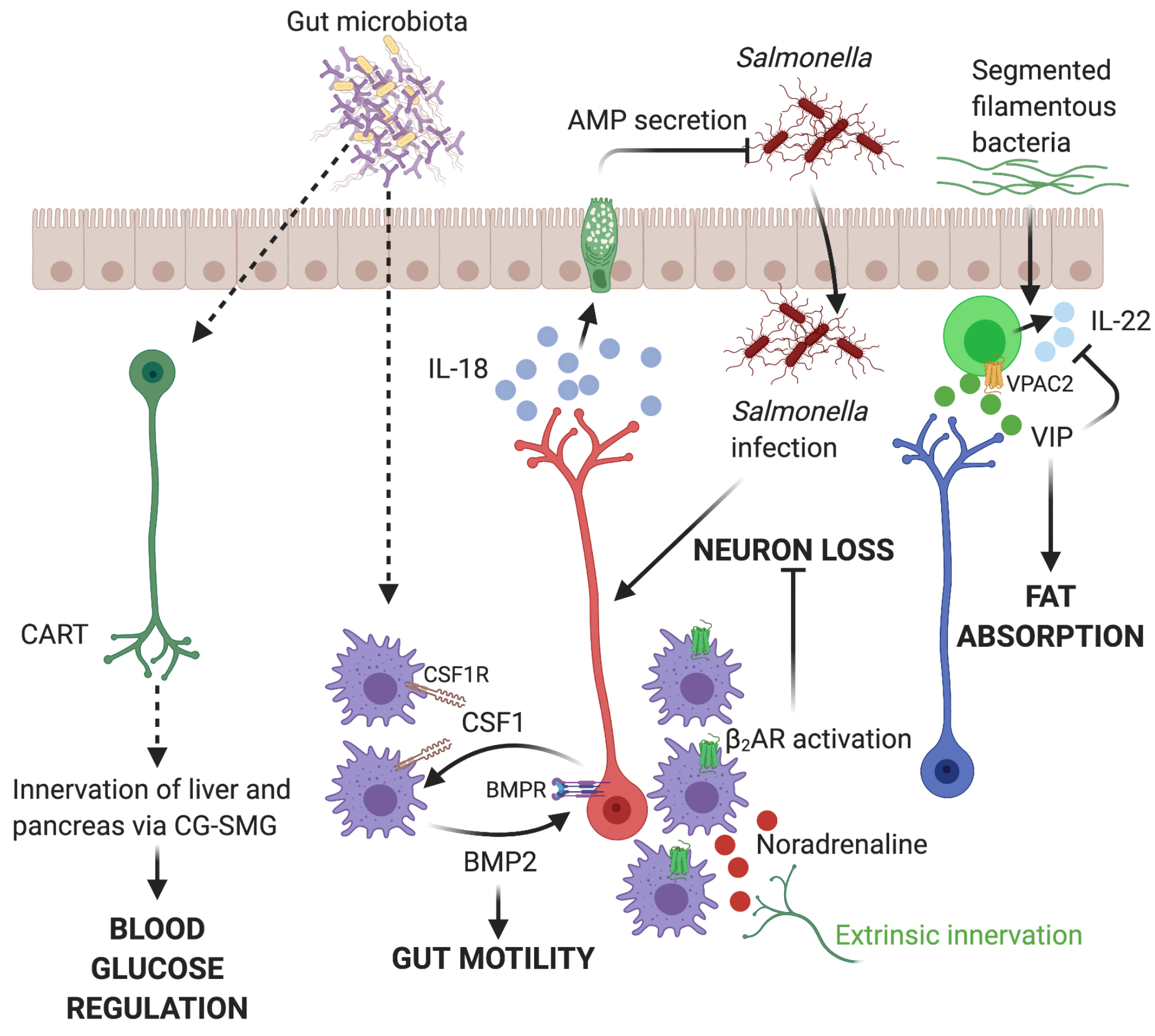

